\title{
Studies of mechanical properties of carbon fabric-polymer hybrid nanocomposite
}

\author{
Dumpala Lingaraju $^{\mathrm{a} *}$, D. Suneel ${ }^{\mathrm{b}}$ and Sree Lakshmi ${ }^{\mathrm{b}}$ \\ ${ }^{a}$ Aditya institute of technology and management(AITAM), Tekkali, A.P, India. and \\ ${ }^{b}$ KL University, A.P, India
}

\begin{abstract}
The development of nanoparticle reinforced composites is presently one of the most explored areas in materials science and engineering. Multiscale composites can be produced with superior properties by combining nanoparticles with traditional reinforcement materials. This research focuses on the development of such composites, through the use of carbon nanofibers (CNFs), carbon fibers and modified epoxy resin for structural and impact applications. Flexural and tensile behavior of composites has been analysed to investigate the effect of modification of epoxy matrix by adding CNFs on the mechanical properties of composites. Better strengths are obtained at $2.5 \mathrm{wt} \%$ of Epoxy terminated butadiene-acrylonitrile copolymer (ETBN) and $2 \mathrm{wt} \%$ of Carbon nanofibers (CNF) in the polymer hybrid nanocomposite. Morphological and fracture analysis of composites were performed by SEM.
\end{abstract}

Keywords: Carbon nanofibers, ETBN, Carbon fabric, Polymer hybrid nanocomposites, Mechanical properties

\section{Introduction}

The development of nanoparticle reinforced composites is presently one of the most explored areas in materials science and engineering. The exceptional properties of nanoparticles have made them a focus of widespread research. Carbon nanotubes (CNTs) carbon nanofibers (CNFs) and other nanoparticles have the potential to greatly enhance the properties of composites when combined with traditional reinforcements such as carbon- glass and aramid-fibers (Gojny et al, 2004).

Carbon nanofibers (CNFs) consist of graphite platelets perfectly arranged in various orientations with respect to the fiber axis giving rise to assorted confirmations (Mordkovich 2003). Carbon nano fibres (CNF) are hollow cylinders with diameters ranging between $100 \mathrm{~nm}-250 \mathrm{~nm}$ and lengths of a few tens of microns giving high aspect ratios (length/diameter $>100)$. They have a larger diameter and are less crystalline, while keeping acceptable mechanical and physical properties. They are expected to be promising nanofiller for the preparation of composites with multiple enhanced properties (Matzek, 2004).

Because of their high aspect ratio and of Vander Waals attractive interactions arising at the nanoscale, CNFs are tangled and form aggregates of different sizes which makes their homogeneous dispersion inside the matrix one of the main hurdles (Maruyama, et al. 2002).

Using ETBN to toughen epoxy resin and then the mechanical property of the toughening epoxy resin casting molding system is studied by Yuan-bo,li et al (Yuan-bo et al., 2010). It shows that with the content of ETBN increasing, the flexural strength, tensile strength and tensile modulus decreased, while the impact strength and elongation at break distinctly increased, which demonstrates that the toughness of the epoxy resin system could be improved effectively by adding ETBN (Yuan-bo et al., 2010).

Yuanxin Zhou et reported that, the tensile, fatigue and fracture tests were performed on unfilled, 1 wt. \%, 2 wt. \% and 3 wt. \% CNF filled SC-15 epoxy to identify the effect of adding CNF on the mechanical properties of epoxy. The highest improvement in strength was obtained with 2 wt. \% loading of CNF. The nanophased epoxy filled with 2 wt.\% CNF was then used with satin weave carbon fabric in a vacuum assisted resin transfer molding (VARTM) set up to fabricate composite panels. Flexural, tensile and fatigue tests were performed to evaluate the effectiveness of CNF addition on the mechanical properties of the composite. The tensile and flexural strengths improved by 11 and $22.3 \%$, 
respectively, compared to the composite without CNF. The fatigue strength was also improved significantly (Yuanxin Zhou et al., 2008).

Epoxy resins are widely used in a variety of applications because of their high chemical and corrosion resistance and good mechanical properties. Carbon nano fibres have been dispersed uniformly into the epoxy resin at a very low concentration $(0.07$ vol. \%). Improvement of $98 \%$ in Young modulus, $24 \%$ in breaking stress and $144 \%$ in work of rupture was achieved in the best sample. The emphasis is on achieving uniform dispersion of carbon nanofibers into epoxy resin using a combination of techniques such as ultra sonication, use of solvent and surfactants (Sohel Rana et al., 2010).

Carbon nanofibers (CNFs) are electrically and thermally conductive and have very good mechanical properties. Research on carbon nano fiber-reinforced nanocomposites has been mainly focusing on carbon nano tube (CNT)-filled nanocomposites. This is due to the fact that CNTs have fewer micro structural defects than CNFs, resulting in better overall properties as well as smaller dimensions and a lower density. However, CNFs are less expensive and can be manufactured at high yields, justifying further in-depth investigation of their impact on nanocomposites (Lan-Hui Sun et al., 2011).

Good dispersion of CNFs leads to an enhancement in both strength and modulus of nanocomposites (Sandler et al. 2002; Ma, et al. 2003; Choi, et al. 2005; Zhou, et al. 2007). Choi et al. (2005) found that CNF/epoxy nanocomposites had a maximum tensile strength and a large Young's modulus with $5 \mathrm{wt} \% \mathrm{CNFs}$, and a reduced fracture strain with increasing filler content.

The mechanical properties of CNFs reinforced phenolic resin matrix composites and carbon/carbon $(\mathrm{C} / \mathrm{C})$ composites were investigated by Shinn-Shyong Tzeng (ShinnShyong Tzeng et al. 2007). The $\mathrm{C} / \mathrm{C}$ composites were obtained by pyrolyzing the phenolic resin composites. For the phenolic resin composites, limited strength enhancement was measured. However, better reinforcing results were obtained for CNF reinforcement as compared to the CNT reinforcement due to the better interfacial bonding. $\mathrm{CNF}$ reinforcement also showed better results for the modulus measurement than those of CNTs. Contrary to the phenolic resin composites, CNTs showed better reinforcing results for $\mathrm{C} / \mathrm{C}$ composites carbonized at 1000 and $1400^{\circ} \mathrm{C}$. For $\mathrm{C} / \mathrm{C}$ composites heat treated at $2400^{\circ} \mathrm{C}$, the flexural strength val- ues were lower than those at 1000 and $1400{ }^{\circ} \mathrm{C}$ and the difference between different reinforcements was not significant. Compared with the flexural strength, the enhancement of flexural modulus was much more significant (ShinnShyong Tzeng et al. 2007).

Epoxy resin is being modified to improve elongation capabilities. Toughness and other mechanical properties of epoxy resins can be significantly improved by adding rubber, in which Epoxy terminated butadiene-acrylonitrile copolymer (ETBN) is conventionally used. In this system, both epoxy groups on ETBN and epoxy groups on epoxy resin to form one network. The present study reports that the flexural and tensile behavior of composites has been analysed to investigate the effect of modification of epoxy matrix by adding ETBN and CNFs of nanocomposites.

\section{Materials used}

Carbon nanofibers (M/s Chemapal Industries, Mumbai) have been used to prepare the nanocomposites. CNFs are having the diameter approximately in the range of $100-200 \mathrm{~nm}$ and length is in the order of $20-30 \mu \mathrm{m}$.

Carbon fabric (T-300, PAN based carbon fabric, Toray, Japan) has been used to make composites.

Epoxy, Diglycidyl Ether of Bisphenol A (DGEBA) and curing agent HY5200 (both supplied by M/s ECMAS Pvt. Ltd, Hyderabad) have been used to process the multi phase nanocomposite.

Epoxy terminated butadiene-acrylonitrile copolymer (ETBN, M/s Fine Finish Organics, Mumbai) has been used to enhance the matrix properties through modification of epoxy by adding ETBN.

\section{Modification of epoxy matrix}

Reactive liquid rubber dissolved in liquid resin, as curing proceeds, rubber precipitates from epoxy and forms fine dispersion of rubber particles with diameter of few micrometres or less. Two compositions were prepared using $2.5 \%$ and $5 \%$ ETBN to modify the epoxy resin. Epoxy was mixed and Di Ethyl Aniline acts as curing agent. Curing was performed at $120{ }^{\circ} \mathrm{C} / 1 \mathrm{~h}$, continued to $150{ }^{\circ} \mathrm{C} / 1 \mathrm{~h}$ and then at $210^{\circ} \mathrm{C} / 3 \mathrm{~h}$. The same procedure is indented for another system to achieve the matrix modification. The morphology and distribution of second phase were carefully observed using optical microscopy and SEM. 


\section{Composites preparation and testing}

Carbon nanofibers were used to understand the effect of nanophase additions on the properties of C-modified epoxy composites. C-modified epoxy and C- modified epoxy/CNFs multiphase nanocomposites have been prepared at $1 \mathrm{wt} \%$ CNFs loading. Initially, CNFs were added directly to the modified epoxy system and then preheated at $60^{\circ} \mathrm{C}$ to reduce the viscosity. This mixture was sonicated with the help of probe type sonicator for $30 \mathrm{~min}$ at below $80^{\circ} \mathrm{C}$. Curing agent was added to the mixture in the ratio $4 \%$ of polymer and $1 \%$ of curing agent by weight to improve the dispersion. Further, this was followed by ball milling at the speed of $150 \mathrm{rpm}$ for 30 min by adding silane coupling agent to the CNF-resin mixture to enhance the degree of dispersion. This mixture was brushed on to the surface of the individual C-fabric layers. Curing was carried out at $120^{\circ} \mathrm{C}$ for $2 \mathrm{~h}$, followed by $180^{\circ} \mathrm{C}$ for $4 \mathrm{~h}$. Thus, Composites were synthesized using modified epoxy resin at 2.5 and $5 \%$ loading of ETBN rubber to modify the epoxy resin and with $1 \mathrm{wt} \%, 2 \mathrm{wt} \%$ and $3 \mathrm{wt} \%$ of CNFs reinforcement.

Three point bend tests were carried out to measure the flexural strength of the polymer hybrid nanocomposites according to ASTM D790. The fracture analysis of failed composites has been analyzed by Environmental Scanning Electron Microscopy (ESEM, FEI, and QUANTA 400) to know the role of CNFs on the strength of nanophased composites. Tensile test was carried out as per ASTM D3039 using Universal testing machine (Dak system INC model UTM machine, india).

\section{Characterization of materials}

Typical SEM image of carbon nanofibers at lower magnification shows presence of agglomerates in the order of few hundred microns as shown in Fig. 1.

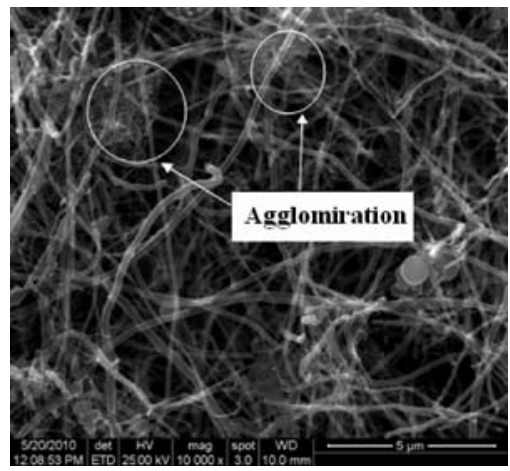

Fig. 1: SEM image of carbon nanofibers showing agglomerates

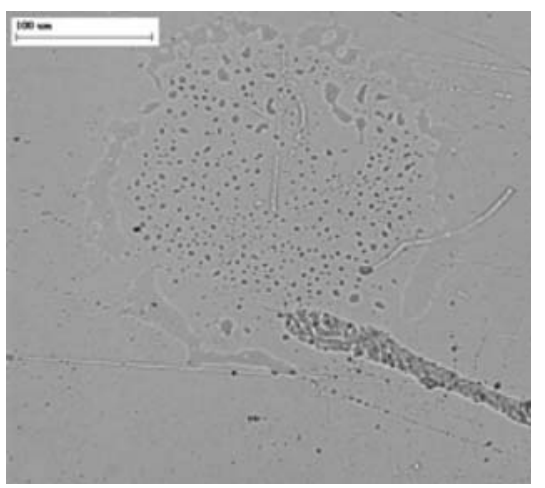

Fig. 2: Optical image of pure epoxy

Optical images of cured pure epoxy resin shows that air bubbles are formed in the sample, as shown in Fig- 2. Optical images of cured Epoxy reinforced with 2.5\% ETBN shows that the dispersion of ETBN (rubbery phase) in epoxy is not uniform (marked by arrows) at lower magnification, as shown in Fig. 3. Typical SEM image of epoxy reinforced with $2.5 \%$ ETBN matrix at lower magnification shows that the dispersion of ETBN is not uniform as shown in Fig. 4.

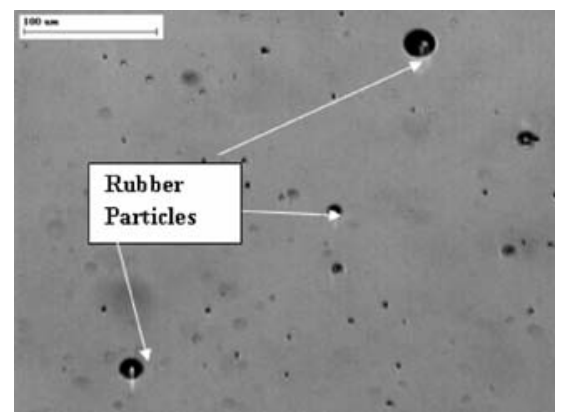

Fig. 3: Optical image of $2.5 \%$ ETBN -Epoxy composite showing dispersion of ETBN

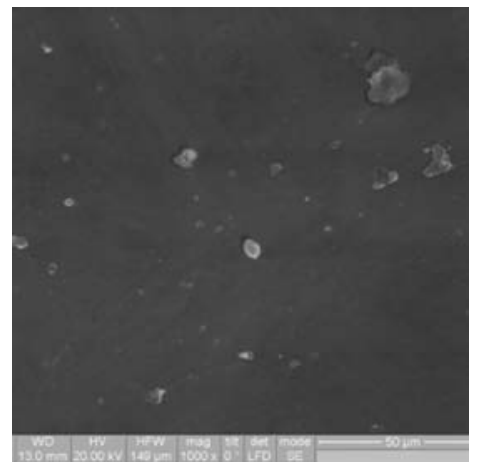

Fig. 4: SEM image of $2.5 \%$ ETBN- epoxy matrix showing rubbery particles

Typical SEM image of $1 \mathrm{wt} \%$ of CNF and $2.5 \mathrm{wt} \%$ of ETBN modified nanocomposite shows presence of clusters, which is due to ETBN phase at lower magnification as shown in 
Fig. 5. The agglomerates are in the order of few microns. At higher magnification, nanosized ETBN particles are observed, which are uniformly distributed in epoxy matrix in Fig. 6. At lower magnification bundles of carbon fibers are observed, as shown in Fig. 7. It can be observed that the nanofibers are formed clusters at most of the regions in Fig. 8. From Fig. 8, carbon nanofibers are entangled more in nature at higher magnification. Variation of size of nanofibers is very high. No alignment of nanofibers is observed at any region in the sample. The distribution of nanofibers shows that the diameter varied significantly. The diameter of nanofibers is in the range of 100-200 $\mathrm{nm}$. However, non uniform distribution of carbon nanofibers is observed from the composite.

From the above observations, it can be concluded that the dispersion of ETBN particles in the epoxy matrix is not uniform. Homogeneous distribution of ETBN phase as well as dispersion of CNFs in the regular epoxy matrix decrease with increasing percentage of ETBN.

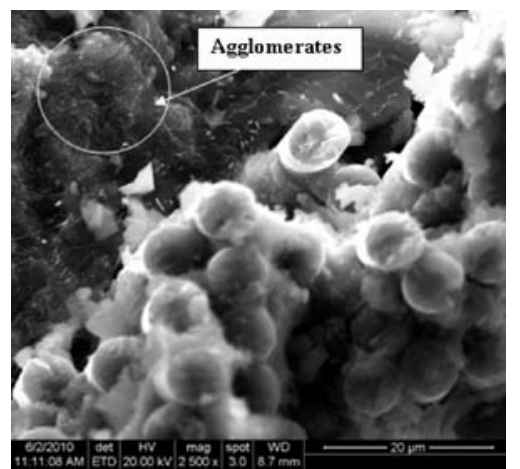

Fig. 5: SEM image of $1 w t \%$ CNF and $2.5 w t \%$ ETBN composite

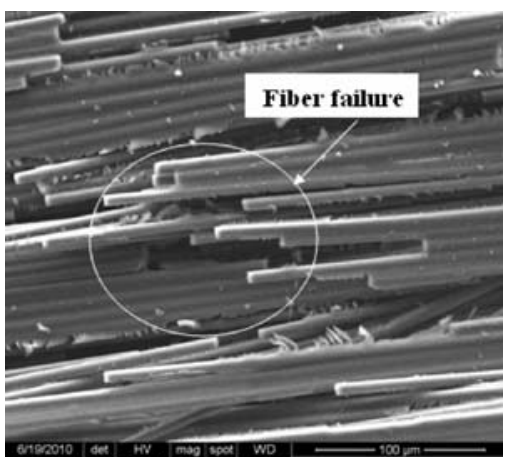

Fig. 6: SEM image of $1 \mathrm{wt} \% \mathrm{CNF}$ and $2.5 \mathrm{wt} \%$ ETBN composite showing nanosized ETBN particles

Typical failure images of $5 \mathrm{wt} \%$ of ETBN modified composite at lower and higher magnifications are shown in Fig. 9 and Fig. 10. Fiber pullout is observed and the fibers are not clean as shown in Fig. 9. The network of ETBN particles in

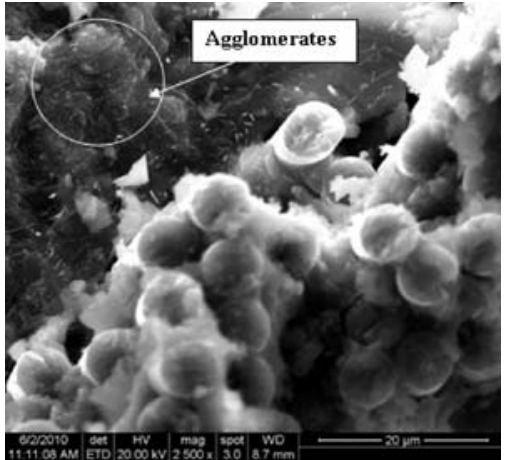

Fig. 7: SEM image of $1 w t \%$ CNF and $2.5 w t \%$ ETBN composite showing agglomerates of CNFs in the matrix

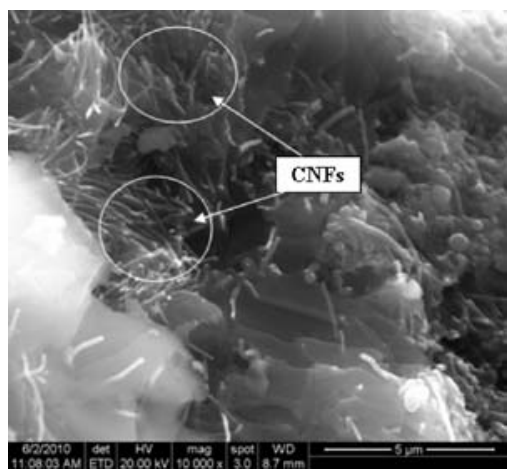

Fig. 8: SEM image of $1 w t \% \mathrm{CNF}$ and $2.5 w t \%$ ETBN composite showing dispersion of CNFs in the matrix

the matrix materials should aid in the transfer of load between the resin and fibers resulting in multiscale reinforc-

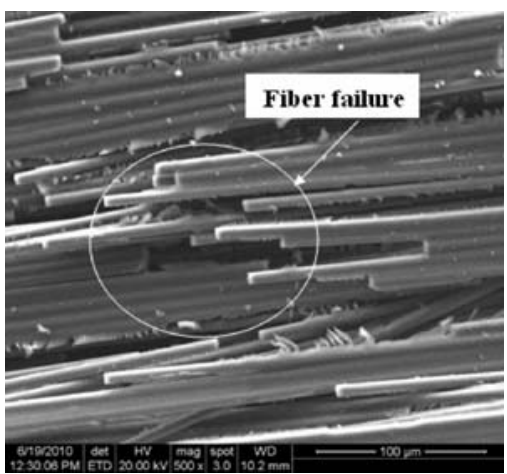

Fig. 9: SEM images of fracture surfaces of $5 w t \%$ of ETBN composite indicating fiber pullout

ing composite With good interfacial bonding and dispersion should enhance the resin dominated properties of the composites, while addition of large quantity of ETBN particles decreases the flexural properties due to formation of strong interfacial bonding with fibers leads to brittle failure. Addition of ETBN phase changes the failure mode of composites more too ductile mode. Bent resin stripes are typical 
evidence of a change in failure mode. Decrease in flexural strength is due to non-uniform dispersion of excess ETBN.

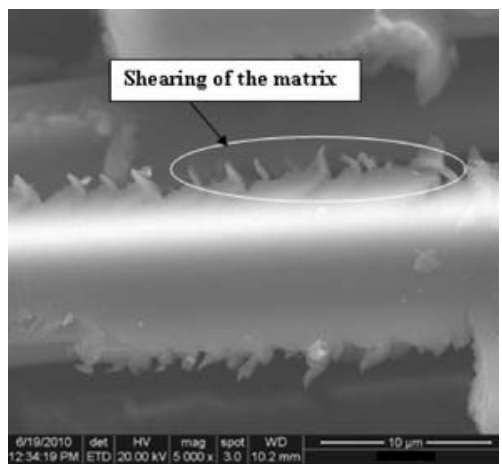

Fig. 10: SEM images of fracture surfaces of $5 w t \%$ of ETBN composite showing plastic deformation of epoxy matrix during loading

At higher magnification, accumulation of ETBN particles in the epoxy matrix is fully covered on the carbon fibers. It may be concluded that the presence of rigid matrix due to modification of epoxy matrix increases the brittleness and gives brittle failure. Fig. 11 and Fig. 12 shows the uniform dispersion of CNFs in the matrix, which indicates the pulling of nanofibers along the loading direction. The increase in tensile strength is may be due to presence of nanofiber pulling from the epoxy matrix.

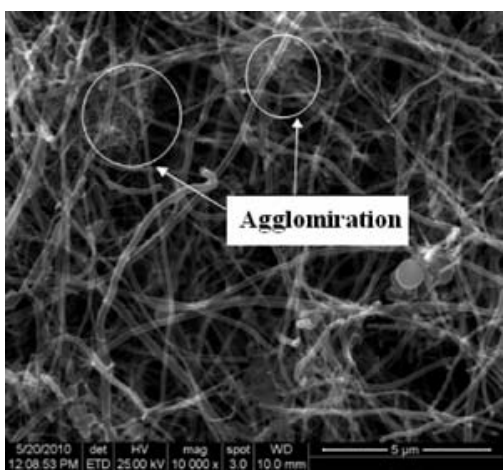

Fig. 11: SEM images of fracture surfaces of $1 \mathrm{wt} \%$ of CNF and $5 w t \%$ of ETBN composite

Mechanical strengths like Tensile strength and Flexural strength are found for the developed hybrid nanocomposites by varying weight percentage of carbon nano fibers and weight percentages of ETBN are reported. Almost all the cases both tensile and flexural strengths are improved with CNF reinforcement and ETBN modification. More than $100 \%$ improvement noted in Tensile strength by reinforcing the CNFs in polymer composites. A constant improvement is noted between $1-2 \mathrm{wt} \%$ of CNF and $2.5 \mathrm{wt} \%$ of ETBN. Maximum of 173 percent of improvement of tensile strength

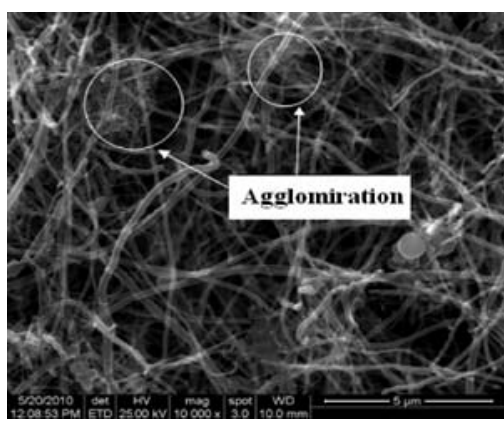

Fig. 12: SEM images of fracture surfaces of $1 w t \%$ of CNF and $5 w t \%$ of ETBN composite

is noted at $1 \mathrm{wt} \%$ of CNF and $5 \mathrm{wt} \%$ of ETBN. Results conclude that $1 \mathrm{wt} \%$ of CNF and $2.5 \mathrm{wt} \%$ of ETBN shows better tensile and flexural strengths. Tensile strength and flexural strength are reported in Fig. 13-15.

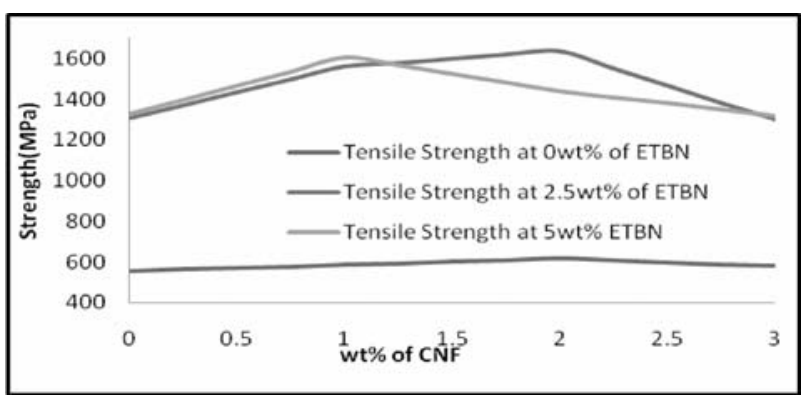

Fig. 13: Tensile strength along with wt $\%$ fraction of CNF and ETBN

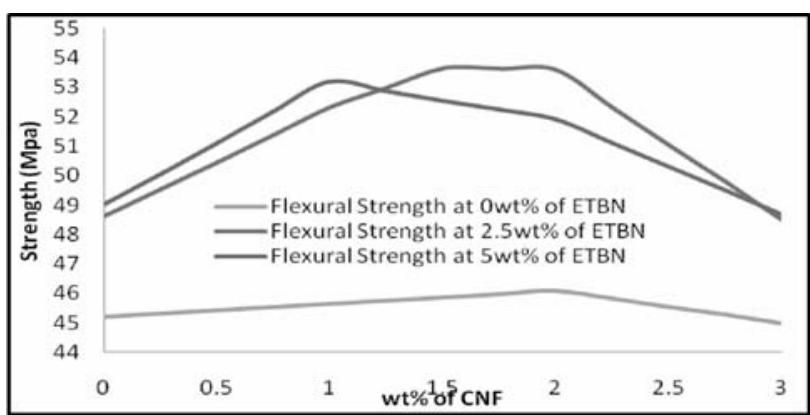

Fig. 14: Flexural strength along with $w t \%$ fraction of CNF and ETBN

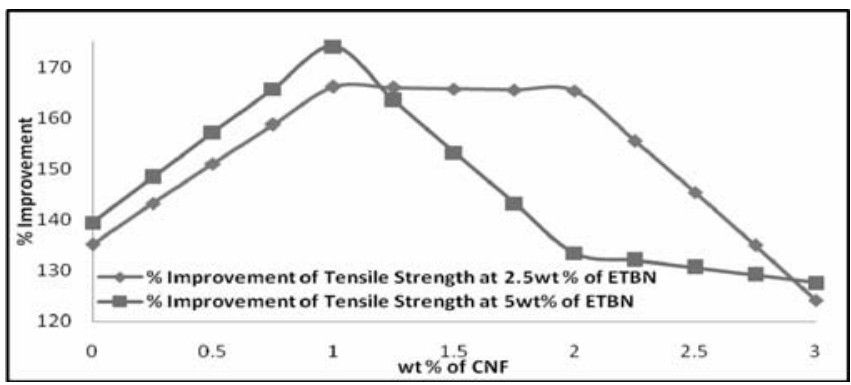

Fig. 15: Percentage Improvement of Tensile strength along with wt\% fraction of CNF and ETBN 


\section{Conclusion}

It is concluded that the modification of epoxy matrix by adding ETBN rubbery phase is achieved as seen by optical and SEM analysis.

From the above observations, it can be concluded that the dispersion of ETBN particles in the epoxy matrix is not uniform at $5 \mathrm{wt} \%$. Homogeneous distribution of ETBN phase as well as dispersion of CNFs in the regular epoxy matrix decreases with increasing percentage of ETBN. It can be concluded that the presence of ETBN phase leads to good interfacial bonding between the matrix and fibers.

The presence of ETBN in the epoxy increases the mechanical properties of the hybrid nanocomposite up to $2.5 \%$ and later it starts decreasing due to the increase of resistance of epoxy matrix. Agglomeration and non-uniform dispersion of CNFs rather than that of uniform dispersion of CNFs and non-uniform growth of rubbery particles of ETBN act as obstacles for the composite failure. Modification of epoxy resin matrix by adding ETBN at higher loading affects the dispersion of nanofibers, which decreases the properties and increases the hardness of epoxy matrix.

It can be suggested that the optimization of ETBN additions can give better enhancement of the mechanical properties of regular epoxy. Lowering the percentage of ETBN below 2.5 $\mathrm{wt} \%$ may gives better dispersion as well as better properties.

\section{References}

Choi YK, Sugimoto KI, Song SM and Endo M 2005. "Mechanical and thermal properties of vapor-grown carbon nanofiber and polycarbonate composite sheets," Materials Letters, 59(27): 3514-3520

Choi YK, Sugimoto KI, Song SM, Gotoh Y, Ohkoshi Y and Endo M 2005. Mechanical and physical properties of epoxy composites reinforced by vapor grown carbon nanofibers, Carbon, 43(10): 2199-2208.

Gojny F, Wichmann M, Kopke U, Fiedler B and Schulte K 2004. Carbon Nanotube-Reinforced Epoxy Composites: Enhanced Stiffness and Fracture Toughness at Low Nanotube Content, Composites Science and Technology, 64: 2363-2371.

Lan-Hui Sun, Zoubeida Ounaies, Xin-Lin Gao, Casey A.Whalen and Zhen-Guo Y 2011. "Preparation, Characterization, and Modeling of Carbon Nanofiber/Epoxy Nanocomposites", Hindawi
Publishing Corporation Journal of Nanomaterials, Article ID 307589, 8 pages.

Ma H, Zeng J, Realff ML, Kumar S and Schiraldi DA 2003. Processing, structure and properties of fibers from polyester/carbon nanofiber composites." Composites Science and Technology, 63(11): 1617-1628.

Mordkovich VZ 2003. Carbon nanofibers: a new ultrahighstrength material for chemical technology, Theoretical Foundations of Chemical Engineering, 37(5): 429-38.

Maruyama B and Alam K 2002. Carbon nanotubes and nanofibers in composite materials, SAMPE Journal, 38(3): 60-69.

Matzek MD 2004. Polymeric carbon nanocomposites: physical properties and osteoblast adhesion Studies, Dayton OH USA, University of Dayton, Master's Thesis.

Sandler J, Werner P, Shaffer MSP, Demchuk V, Altstadt V and Windle $\mathrm{AH}$ 2002. Carbon-nanofibre-reinforced poly (ether ether ketone) composites, Composites Part A, 33(8): 1033-1039.

Shinn-Shyong Tzeng and Yu-Hun Lin 2007.Mechanical properties of carbon-carbon composites reinforced with carbon nanotubes or carbon nanofibers, 16th international conference on composite materials, p: 1-5.

Sohel R, Alagirusamy R. and Mangala J 2010. Journal of Applied Polymer Science, 118(4): 2276-2283.

Yuan-bo WU, Gang LI, HUANG Zhi-bin and Peng L. I. (2010). "Study on the structure and property of ETBN toughen epoxy resin", DOI: CNKI:SUN: BLGF. 0.2010-05-011

Yuanxin Z, Farhana P, Shaik J and Mallick P K 2008. Improvement in mechanical properties of carbon fabric-epoxy composite using carbon nanofibers, Journal of Materials Processing Technology, 198(1-3): 445453.

Zhou Y, Pervin F and Jeelani S 2007. Effect vapor grown carbon nanofiber on thermal and mechanical properties of epoxy, Journal of Materials Science, 42(17): 75447553.

Manuscript received on 18 April 2011; revised on 14 September 2011; accepted on 15 November 2011. 